\title{
Intestinal alkaline phosphatase targets the gut barrier to prevent aging
}

Florian Kühn, ${ }^{1,2}$ Fatemeh Adiliaghdam, ${ }^{1}$ Paul M. Cavallaro, ${ }^{1}$ Sulaiman R. Hamarneh, ${ }^{1}$ Amy Tsurumi, Raza S. Hoda, ${ }^{3}$ Alexander R. Munoz, ${ }^{1}$ Yashoda Dhole, ${ }^{1}$ Juan M. Ramirez, ${ }^{1}$ Enyu Liu, ${ }^{1}$ Robin Vasan, ${ }^{1}$ Yang Liu, ${ }^{1}$ Ehsan Samarbafzadeh, ${ }^{1}$ Rocio A. Nunez, ${ }^{1}$ Matthew Z. Farber, ${ }^{1}$ Vanita Chopra, ${ }^{4}$ Madhu S. Malo, ${ }^{1}$ Laurence G. Rahme, ${ }^{1,5,6}$ and Richard A. Hodin ${ }^{1}$

'Department of Surgery, Massachusetts General Hospital (MCH), Harvard Medical School, Boston, Massachusetts, USA. ${ }^{2}$ Department of General, Visceral and Transplant Surgery, Hospital of the University of Munich, Munich, Germany. ${ }^{3}$ Department of Pathology and ${ }^{4}$ Department of Neurology, MGH, Harvard Medical School, Boston, Massachusetts, USA. ${ }^{5}$ Shriners Hospital for Children, Boston, Massachusetts, USA. ${ }^{6}$ Department of Microbiology and Immunobiology, Harvard Medical School, Boston, Massachusetts, USA.

Gut barrier dysfunction and gut-derived chronic inflammation play crucial roles in human aging. The gut brush border enzyme intestinal alkaline phosphatase (IAP) functions to inhibit inflammatory mediators and also appears to be an important positive regulator of gut barrier function and microbial homeostasis. We hypothesized that this enzyme could play a critical role in regulating the aging process. We tested the role of several IAP functions for prevention of age-dependent alterations in intestinal homeostasis by employing different loss-of-function and supplementation approaches. In mice, there is an age-related increase in gut permeability that is accompanied by increases in gut-derived portal venous and systemic inflammation. All these phenotypes were significantly more pronounced in IAP-deficient animals. Oral IAP supplementation significantly decreased age-related gut permeability and gut-derived systemic inflammation, resulted in less frailty, and extended lifespan. Furthermore, IAP supplementation was associated with preserving the homeostasis of gut microbiota during aging. These effects of IAP were also evident in a second model system, Drosophilae melanogaster. IAP appears to preserve intestinal homeostasis in aging by targeting crucial intestinal alterations, including gut barrier dysfunction, dysbiosis, and endotoxemia. Oral IAP supplementation may represent a novel therapy to counteract the chronic inflammatory state leading to frailty and age-related diseases in humans.

Authorship note: FK and FA are cofirst authors.

Conflict of interest: The authors have declared that no conflict of interest exists.

Copyright: (c) 2020, American Society for Clinical Investigation.

Submitted: October 10, 2019 Accepted: February 20, 2020 Published: March 26, 2020.

Reference information: JCI Insight. 2020;5(6):e134049.

https://doi.org/10.1172/jci.

insight.134049.

\section{Introduction}

Intestinal homeostasis appears to be a determinant factor for healthy aging (1-3). An increased gut permeability and chronic low-grade inflammation linked to persistent gut-derived endotoxemia play crucial roles in a variety of age-related diseases (1-5). Moreover, age-associated compositional changes of the gut microbiota seem to interact with several physiological transitions and pathologies (1-5). Treatments targeting these age-related alterations are considered as potential approaches to prevent pathogenic deterioration and frailty (1-4). However, reliable interventions specifically targeting gut barrier function and microbial composition are currently very limited (5).

The gut brush border enzyme intestinal alkaline phosphatase (IAP) is expressed in the enterocytes of the proximal small intestine, and its activity is known to decrease with age $(6,7)$. Many of the functions of IAP have recently been linked to aging and inflammation; IAP is an antiinflammatory factor that detoxifies a variety of bacterially derived proinflammatory factors such as LPS, CpG-DNA, and flagellin (8). In addition, IAP promotes the gut barrier function, likely through the upregulation of intestinal tight junction proteins $(9,10)$. IAP has also been shown to promote growth of intestinal commensal bacteria, preserving the healthy homeostasis of the gut microbiota $(11,12)$. The antiinflammatory functions of IAP have already been tested in a few clinical trials showing its efficacy (e.g., in blocking endotoxemia in septic patients and flares in patients with ulcerative colitis). As a naturally 
occurring enzyme, IAP has a low-risk profile and can be supplemented orally or via i.v. administration $(13,14)$. Based upon the functional roles for IAP (in particular, its salutary role on gut barrier integrity and its ability to inhibit bacteria-derived inflammatory mediators), we hypothesized that this enzyme could play a critical role in regulating the aging process and that IAP supplementation might counteract the chronic inflammatory state leading to age-related frailty and disease.

\section{Results}

An age-dependent decline of IAP activity is paralleled by increased gut permeability and systemic inflammation. To determine if IAP might play a role in human aging, we first tested IAP activity in ileal contents from 60 stoma patients of different ages (Supplemental Table 1; supplemental material available online with this article; https://doi.org/10.1172/jci.insight.134049DS1). We found a significant decline in IAP activity with age (Figure 1A). Before starting animal experiments, we also confirmed that IAP activity similarly decreases with age in mice. IAP activity was measured in stool and ileal content of young (4-month-old) and old (21-month-old) WT C57BL/6J mice by IAP assay. IAP activity was significantly lower in both the ileal fluid and the stool of old mice (Figure 1B). Because IAP is known to promote gut barrier function, and because impairment of the intestinal barrier is thought to play a critical role in the aging process, we measured gut permeability in IAP-KO and WT mice of different age groups. The FITC-dextran test showed an age-dependent increase in gut permeability, significantly influenced by IAP deficiency (Figure 1C). Furthermore, expression levels of intestinal tight junction proteins were measured in ileal tissue of IAP-KO and WT animals. Again, age and loss of IAP were associated with a significant reduction in expression levels of Occludin and ZO-1 (Figure 1, D and E). Gut hyperpermeability is known to contribute to endotoxemia and local and systemic inflammation. Therefore, proinflammatory cytokines and endotoxins were measured in ileal tissue and serum of IAP-KO and WT mice of different ages. Inflammatory parameters and systemic endotoxin levels were significantly higher in older mice and in animals lacking IAP (Figure 1, F-I).

Lack of IAP is associated with more severe aging-related liver inflammation, steatosis, and increased proinflammatory characteristics of portal serum in mice. We next determined if there is an age-dependent increase in the expression levels of the aging-associated, proinflammatory cytokines IL- 6 and TNF in liver tissue. A significant increase in expression levels of both cytokines in liver tissue of WT mice was observed as a function of age (Figure 2, A and B). IAP-deficiency was associated with significantly increased expression levels of these 2 cytokines (Figure $2, \mathrm{~A}$ and $\mathrm{B}$ ).

To examine age-related histologic changes and investigate the possible effect of IAP in such alterations, the liver, small intestine, and colon of young and old, WT, and IAP-KO mice were compared. For liver tissue, H\&E and Oil Red O staining were performed. Within the liver, hepatocyte vacuolation, ballooning degeneration, and inflammation, infiltration of predominantly lymphocytes, and scattered neutrophils were increased in old compared with the young WT mice. Furthermore, these changes in the liver were seen to a greater extent in aged IAP-KO mice. Marked microvesicular and macrovesicular steatosis were noted in both the 21-month-old WT and IAP-KO mice; however, more macrovesicular deposits were seen in the IAP-KO mice. Accordingly, the macrosteatosis score increased with age and was significantly higher in IAP-KO than in WT mice (Figure 2C). The Oil Red O staining illustrates the marked differences in neutral triglyceride and lipid deposits when young and old WT and IAP-KO mice were compared (Figure 2D). In contrast to the differences seen in the liver, histological comparison between intestinal tissues (ileum and colon) of different ages and genotypes revealed a similar normal appearance (images not shown).

It is well established that gut-derived proinflammatory mediators contribute to liver inflammation. To investigate the impact of IAP on endotoxin dissemination, we measured endotoxin levels in systemic and portal serum from young and old WT and IAP-KO mice by limulus amebocyte lysate (LAL) assay. The amount of LPS in portal and systemic serum (Figure 1I and Figure 2E) increased significantly as a function of age but was $>1000$ times higher in portal compared with systemic serum, regardless of age or genotype, consistent with its gut source. The absence of IAP was associated with significantly more LPS in both portal and systemic blood (Figure 1I and Figure 2E). To further determine the proinflammatory characteristics of portal and systemic serum from young and old IAP-KO and WT mice, we investigated the inflammatory response of target cells exposed to various sera. For this, we incubated primary mouse BM-derived macrophages with portal and systemic serum from young and old WT and IAP-KO mice for 24 hours and measured Tnfa mRNA levels in the targeted cells (Figure $2 \mathrm{~F})$. Upon incubation of target cells, we found that both systemic and portal serum from old animals 
A

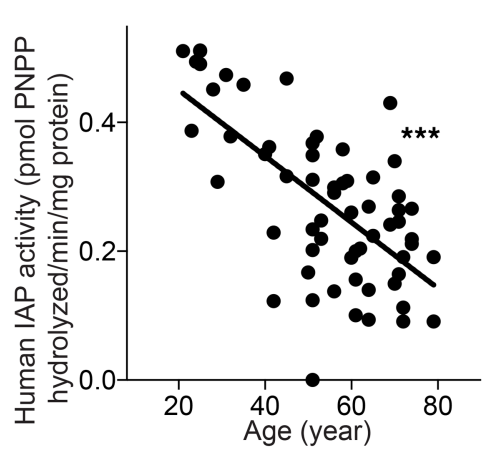

D

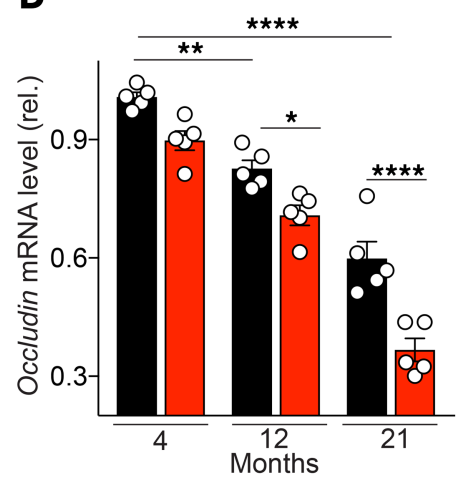

G

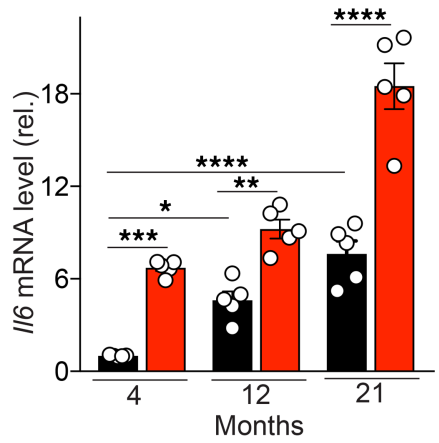

B

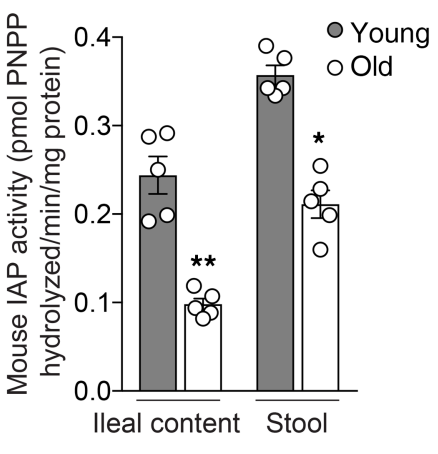

$\mathbf{E}$

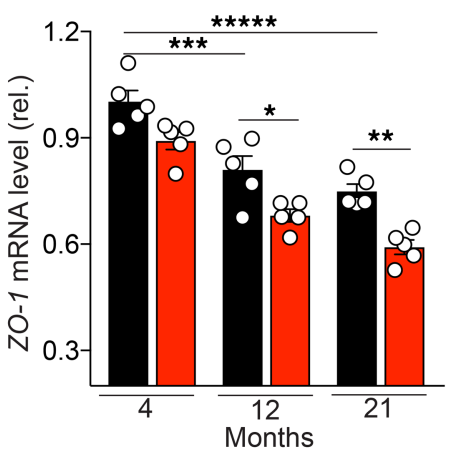

H

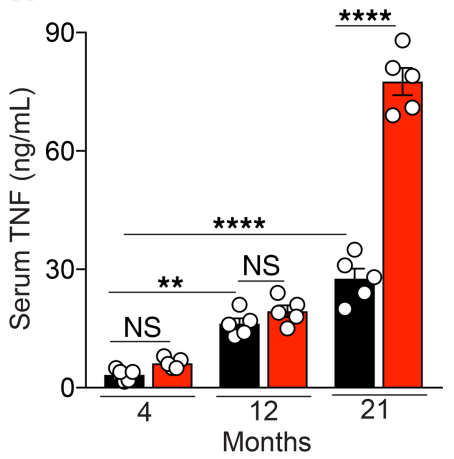

C

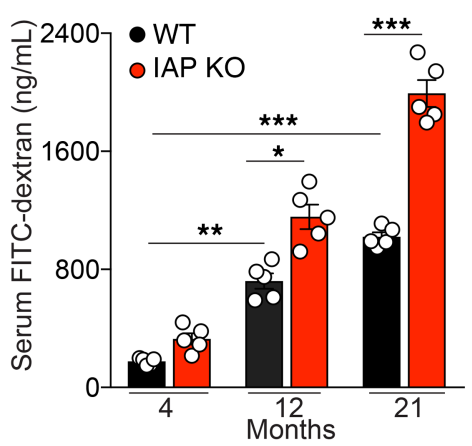

$\mathbf{F}$

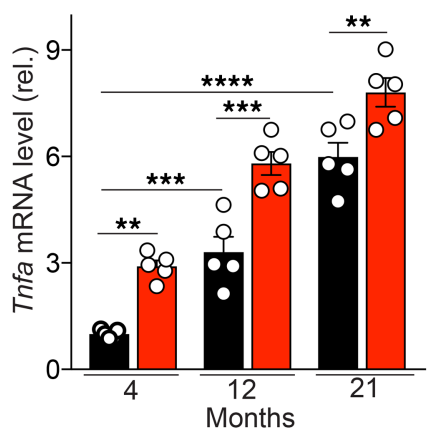

I

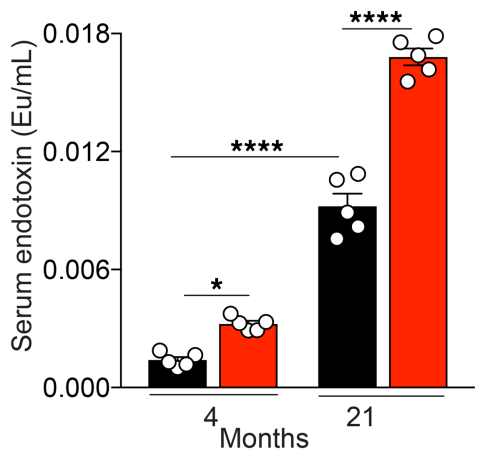

Figure 1. The age-dependent decline of IAP activity is paralleled by gut barrier dysfunction and systemic inflammation. (A and B) IAP activity in human ileal contents $(n=60)(\mathbf{A})$ and stool and ileal content of young and old WT mice (B) measured by pNPP assay. (C) Gut permeability measured by FITC-dextran. (D and E) Ileal tight junction proteins Occludin and ZO1 mRNA levels, normalized by Bactin and measured by qPCR. (F and G) Ileal Tnfa and II6 mRNA levels measured by qPCR. (H) Systemic serum TNF level measured by ELISA. (I) Systemic serum endotoxin level measured by limulus amebocyte lysate (LAL) assay. Each group included 5 animals, and data are representative of 3 biological replicates. Comparisons were made using Pearson's correlation analysis, unpaired Student $t$ tests, or ANOVA with multiple-comparisons post hoc tests (Tukey's HSD). ${ }^{*} P<0.05$, ${ }^{*} P<0.01,{ }^{* * *} P<0.001,{ }^{* * *} P<0.0001$.

induced a significantly higher inflammatory response than serum derived from young animals. We also detected a significant difference between the magnitude of Tnfa expression induced by portal compared with systemic serum. Finally, portal serum from IAP-KO mice resulted in a clearly more pronounced inflammatory response than serum from their WT counterparts.

Long-term IAP supplementation leads to reduced frailty and increased lifespan in mice. Given the impact of IAP deficiency on gut barrier dysfunction, endotoxemia, and inflammation during aging, we evaluated frailty and lifespan in IAP-KO mice, WT control mice (vehicle), and mice receiving IAP supplementation. IAP supplementation was started at the age of 10 months and, as expected, led to significantly higher stool IAP activity levels (Supplemental Figure 1). We found that IAP deficiency was associated with more frailty and a shorter lifespan compared with their WT littermates (Figure 3, A and B). At birth, IAP-deficient mice did not show any visible defects and were indistinguishable from their WT littermates. 
A

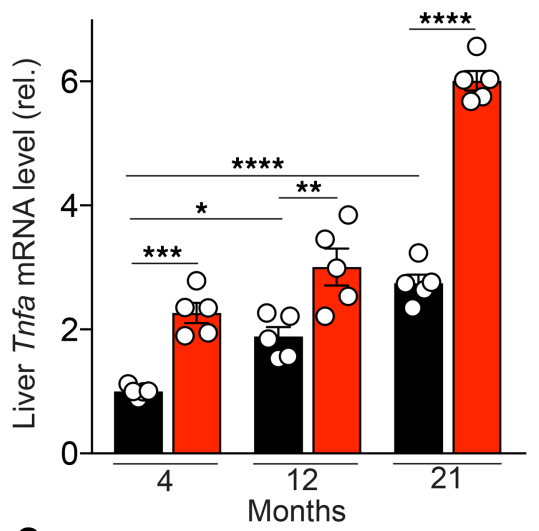

C

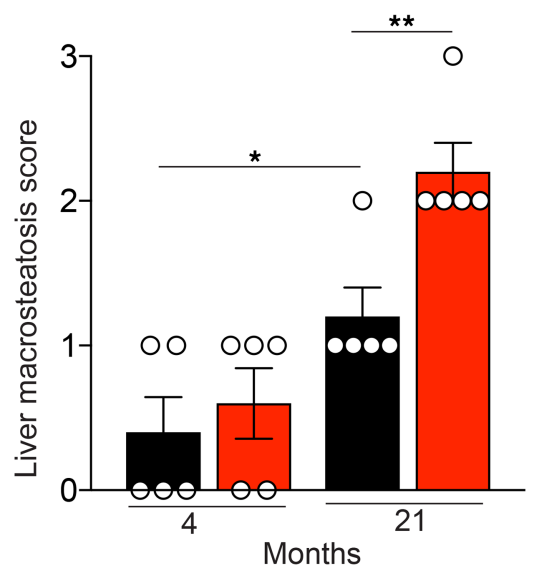

E

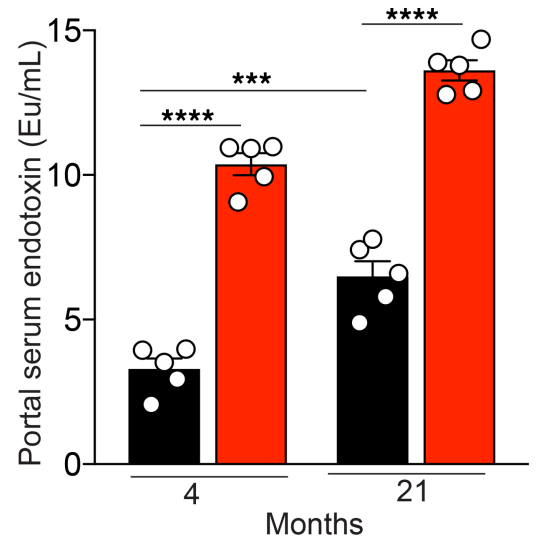

B
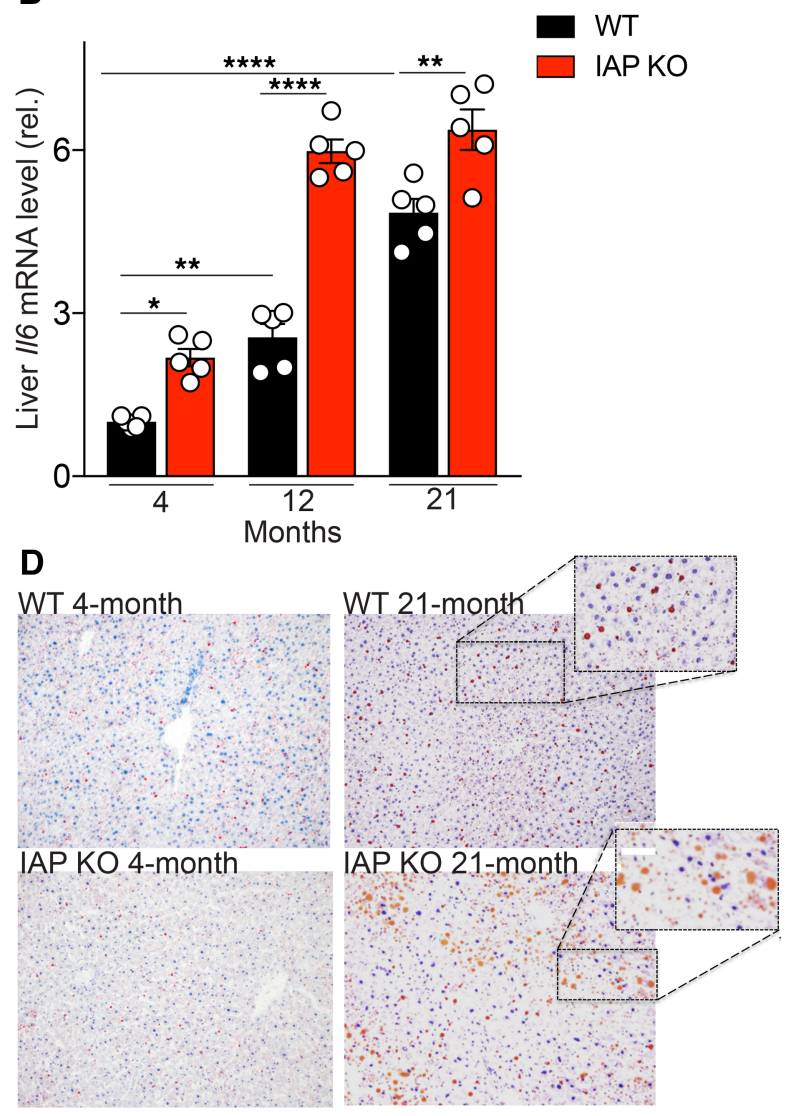

F

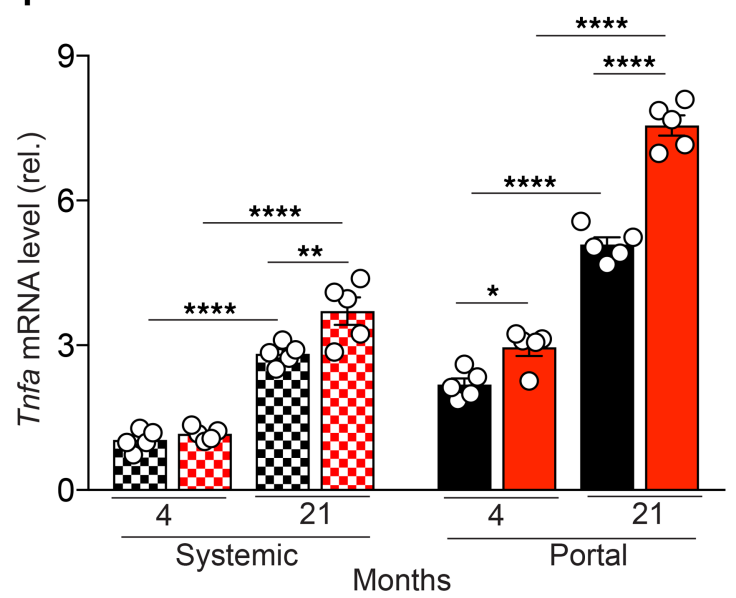

Figure 2. Lack of IAP is associated with a severe aging-related liver inflammation and an increased proinflammatory characteristic of portal serum. (A and B) Liver Tnfa and II6 mRNA levels measured by qPCR. (C) Liver macrosteatosis score (Macrosteatosis 0\%-5\%, grade 0; 5\%-33\%, grade 1; 33\%-66\%, grade 2; $66 \%-100 \%$, grade 3). (D) Oil Red 0 staining of liver (magnification, 20x). (E) Portal serum endotoxin level measured by LAL assay. (F) Tnfa mRNA level of primary mouse BM-derived macrophages incubated with defined systemic or portal serum for 24 hours. Each group included 5 animals, and data are representative of 3 biological replicates. ${ }^{*} P<0.05,{ }^{* *} P<0.01,{ }^{* *} P<0.001,{ }^{* * *} P<0.0001$, as measured by ANOVA with multiple-comparisons post hoc tests (Tukey's HSD).

In addition, during the course of their lives, IAP-KO mice did not develop any significant gross abnormalities in appearance or fertility, including after breeding through multiple generations. No significant differences in frailty index existed among mice younger than 12 months (Figure 3B). In contrast, when we quantified frailty in aged mice, significant differences were observed in older mice ( $>12$ months), and IAP deficiency was associated with a significant difference in frailty (Figure 3B). There was no difference in body weight of IAP-KO and WT mice (age 4-21 months) when they were fed a standard chow diet 
A

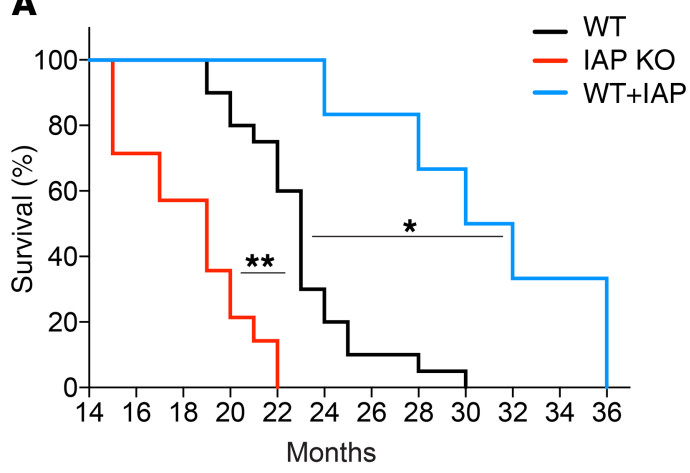

Figure 3. IAP supplementation extends lifespan in mice. (A) Survival of IAP-KO, WT, and IAP-supplemented mice (20 WT, 14 IAP-KO, and 6 IAP-supplemented mice). (B and C) Clinical frailty index of WT and IAP-KO mice (B) and vehicle- or IAP-supplemented WT mice (C). Unpaired Student $t$ tests or ANOVA with multiplecomparisons post hoc tests (Tukey's HSD) were used as statistical tests. Survival data were compared using the log-rank significance test. ${ }^{*} P<0.05,{ }^{* *} P<0.01$.
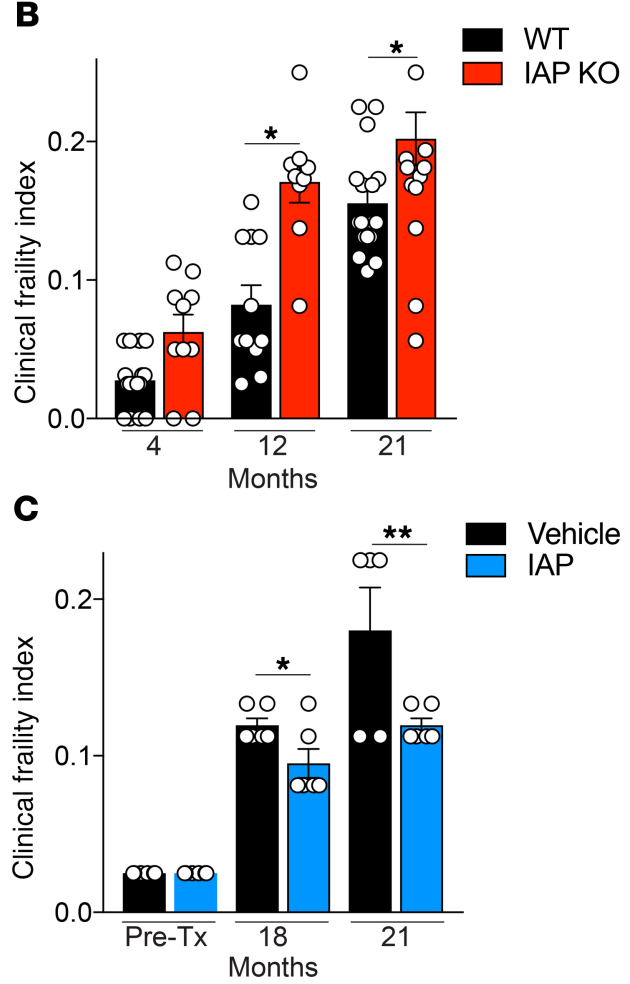

(Supplemental Figure 1). There was also no difference in the frailty index of WT mice before starting IAP or vehicle supplementation (Figure 3C). After 8 and 11 months, IAP supplementation led to marked differences in frailty; higher intestinal IAP activity levels were associated with lower frailty indices after 8 and 11 months of IAP compared with vehicle supplementation (Figure 3C). Moreover, IAP-KO mice had a shortened lifespan, whereas IAP supplementation led to a significantly extended lifespan compared with mice receiving vehicle alone (Figure $3 \mathrm{~A}$ ).

Long-term IAP supplementation ameliorates aging-induced gut barrier dysfunction, endotoxemia, and chronic inflammation in mice. To further explore the effects of IAP supplementation on intestinal alterations in aging, we measured gut permeability by a systemic serum FITC-dextran test in 21-month-old WT mice that had received IAP supplementation for 11 months. Gut permeability was significantly reduced in mice supplemented with IAP, compared with control animals (Figure 4A). Next, blood serum endotoxin and cytokine levels in WT mice receiving IAP or vehicle were measured. Long-term IAP supplementation led to a significant reduction in endotoxin and proinflammatory cytokine levels compared with control mice (Figure 4, B-E). Furthermore, fecal Lipocalin 2 (Lcn2) levels, a sensitive marker for chronic (low-grade) intestinal inflammation, were measured in the stool of WT mice with or without IAP supplementation (Figure 4F). Mice receiving IAP had significantly lower Lcn2, as well as fecal endotoxin levels (Figure 4, F and G).

Long-term IAP supplementation leads to an improved metabolic profile in WT mice. Given the IAP effects on gut barrier function, endotoxemia, and systemic inflammation, we next examined the impact of IAP 
A

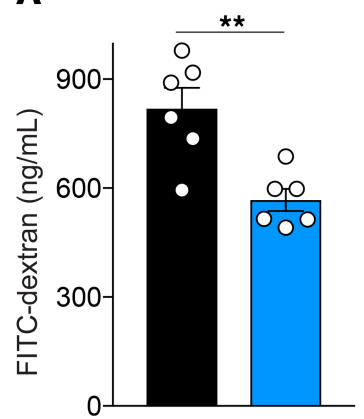

C

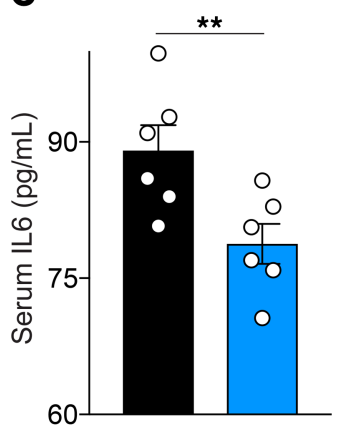

$\mathbf{F}$

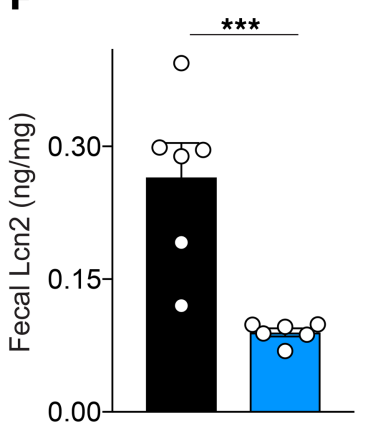

B

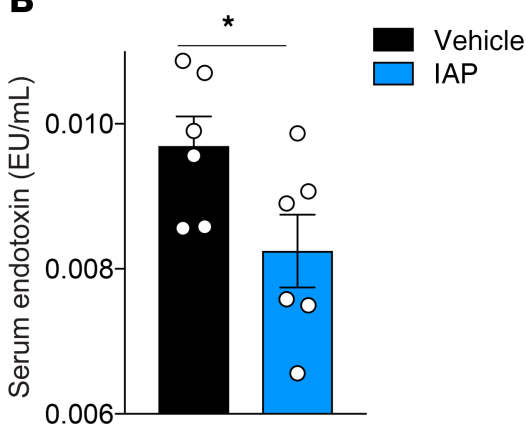

$\mathbf{E}$

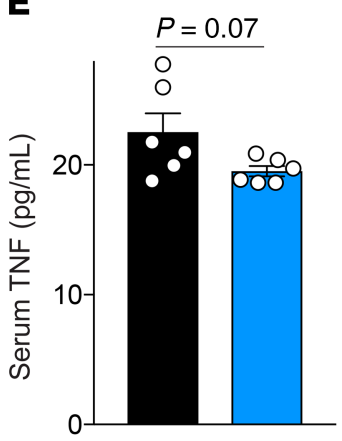

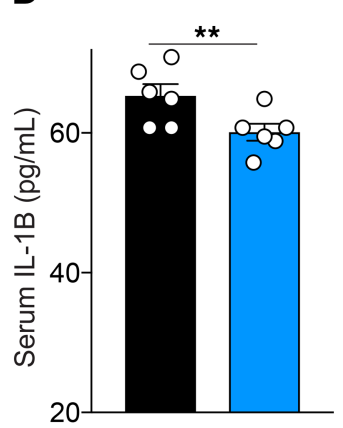

G

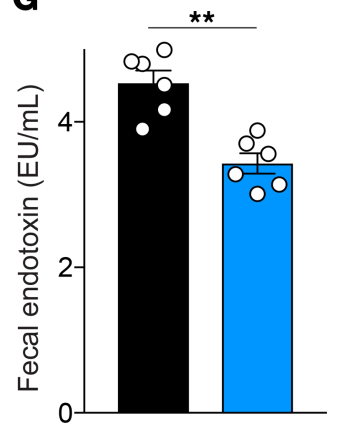

Figure 4. Long-term IAP supplementation ameliorates aging-induced gut barrier dysfunction and chronic systemic inflammation. (A) Gut permeability measured by systemic serum FITC-dextran 4 hours after oral gavage in 18-monthold WT mice supplemented with vehicle or IAP for 8 months. (B) Blood serum endotoxin levels in WT vehicle- or IAP-supplemented mice measured by LAL assay. (C-E) Systemic serum IL-6, IL-1B and TNF- $\alpha$ levels in vehicle- or IAP-supplemented mice measured by ELISA. (F) Fecal Lipocalin 2 (Lcn2) levels measured by ELISA. (G) Fecal endotoxin levels measured by LAL assay. Each group included 6 animals. Data expressed as mean \pm SEM. Unpaired Student $t$ tests are used as statistical tests. ${ }^{*} P<0.05,{ }^{* *} P<0.01,{ }^{* *} P<0.001$.

treatment on the metabolic profile in aging mice. As expected from earlier published data from our lab (15) in which IAP was effective in preventing metabolic syndrome in mice fed a high-fat diet, long-term IAP treatment in mice receiving a standard chow diet was associated with a significantly improved lipid profile (Figure 5, A-D), as well as lower blood glucose and urea nitrogen levels (Figure 5, E and F). Furthermore, IAP treatment led to lower serum liver enzymes (Figure $5, \mathrm{G}$ and $\mathrm{H}$ ).

Long-term IAP supplementation prevents aging-related compositional changes of gut microbiota. To determine the effect of supplemental oral IAP on age-associated changes in commensal bacterial populations, we performed 16S rRNA sequencing and analysis of fecal samples at various time points (before treatment, 1 month, and 6 months after treatment). As measured by observed operational taxonomic units (OTUs) and Shannon diversity index, $\alpha$ diversity did not change over time for either vehicle-treated or IAP-treated mice. Principal Component Analysis (PCA) on relative abundance of phyla at various time points and across treatment groups was performed. The PCA demonstrated that the first principal component, explaining $37.8 \%$ of the variation, separated the control group of vehicle-treated mice at the 6-month time point (Figure 6, A and B). The fecal samples from pretreatment, 1 month of treatment, and 6 months of IAP treatment mice all 
A

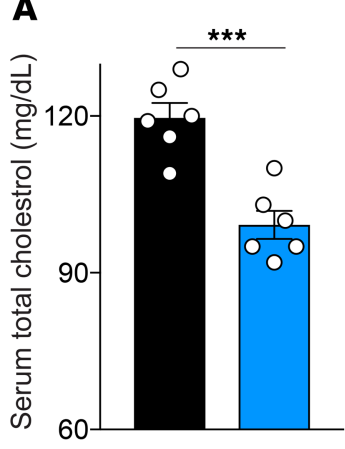

D

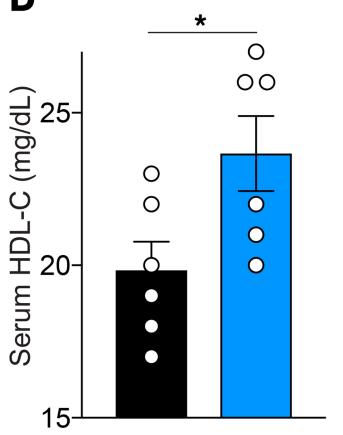

B

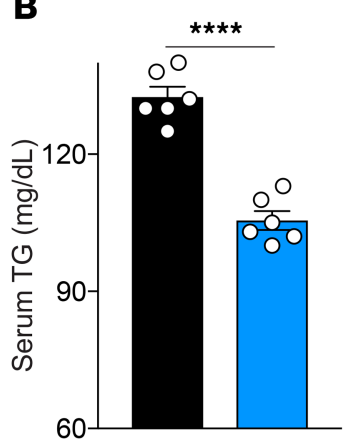

E

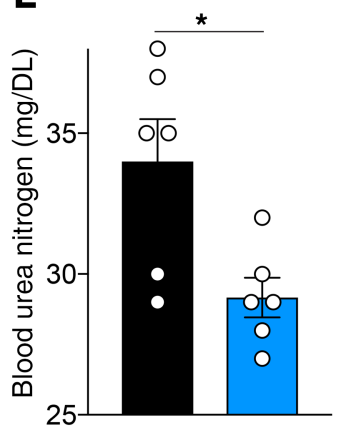

C

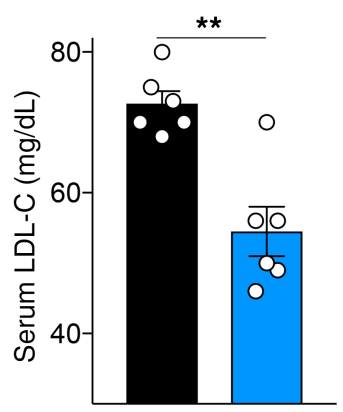

$\mathbf{F}$

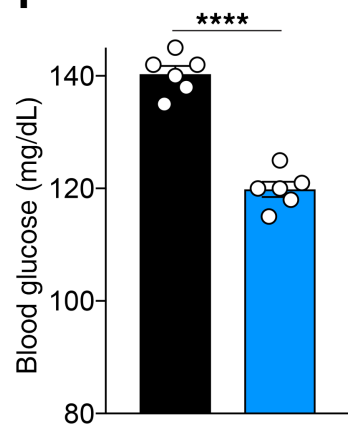

G

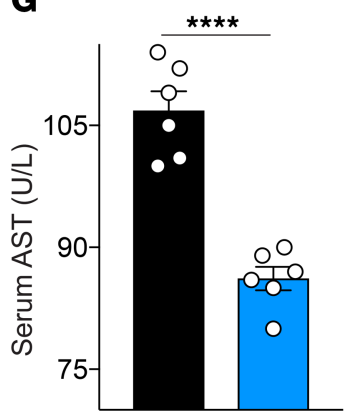

H

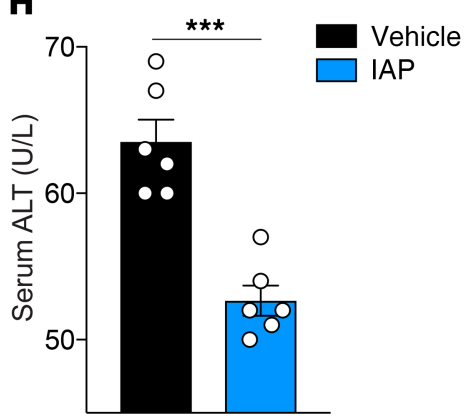

Figure 5. Long-term IAP supplementation leads to an improved metabolic profile in aging mice. (A) Serum total cholesterol levels in 18-month-old WT mice supplemented with vehicle or IAP for 8 months. (B) Serum triglyceride levels. (C) Serum LDL-C levels. (D) Serum HDL-C levels. (E) Blood urea nitrogen levels. (F) Blood glucose levels. (G and H) Serum liver enzyme levels (AST, ALT). Each group included 6 animals. Data expressed as mean \pm SEM. Two-tailed unpaired Student's $t$ tests were used. ${ }^{*} P<0.05,{ }^{* *} P<0.01,{ }^{* *} P<0.001,{ }^{* * *} P<0.0001$.

clustered together, indicating overall similarity in the phylum composition among these groups. With regard to the relative abundance of specific phyla, we observed that control mice demonstrated a clear change in phyla among control mice over the 6-month experiment. Specifically, a statistically significant decrease was observed in the abundances of Proteobacteria, Actinobacteria, Epsilonbacteraeota, and Deferribacteres (Figure 6, C-F), while Tenericutes and Verrucomicrobia abundance was significantly increased (Figure 6, $\mathrm{G}$ and $\mathrm{H}$ ). No significant change was detected for the 2 most common phyla, Bacteroidetes and Firmicutes. In contrast, IAP-treated mice displayed minimal change in the relative abundance of phyla over time, with only a marginal increase in the abundance of Tenericutes at the 1-month time point, which reverted to pretreatment abundance after 6 months (Figure 6G). When comparing the relative abundance of phyla between IAP- and control-treated mice after 6 months, the abundance of the 2 most common phyla were similar; however, vehicle-treated mice had significantly less Proteobacteria, Actinobacteria, Epsilonbactareota, and Deferribacteres and significantly more Verrucomicrobia. Taken together, our data demonstrate that longterm IAP treatment appears to prevent the age-associated change in fecal microbial composition over time.

IAP supplementation improves physical performance and survival in Drosophilae. Given the above findings in mice, we chose to confirm our findings employing Drosophila melanogaster, another commonly used model 


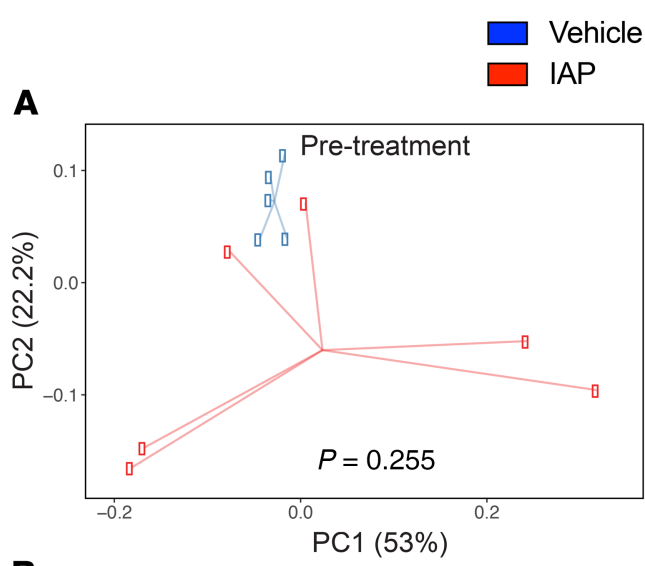

B

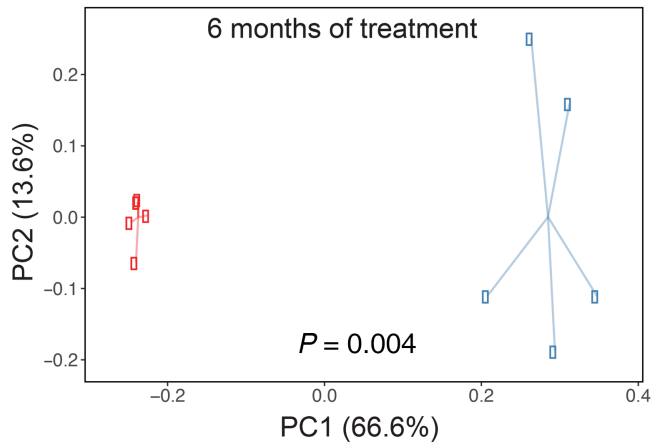

C

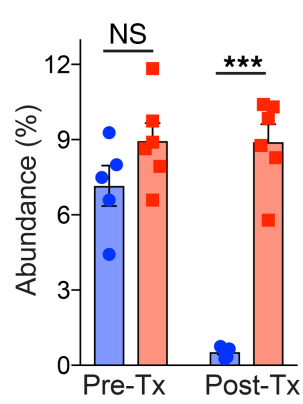

E Epsilonbacteraeota

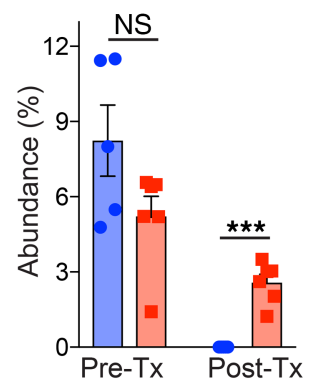

G

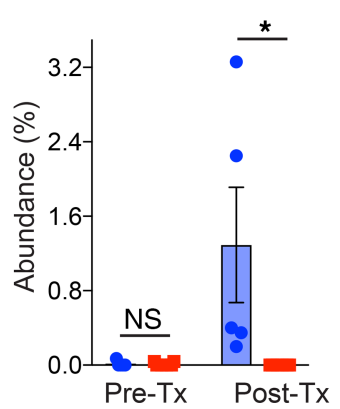

D Actinobacteria

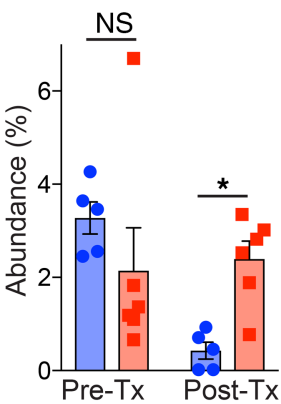

F Deferribacteres

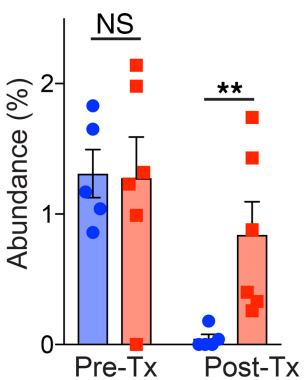

H Verrucomicrobia

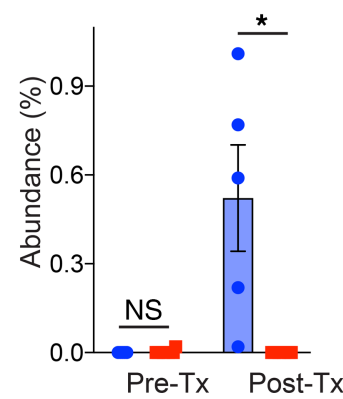

Figure 6. Long-term IAP supplementation prevents age-induced microbiome dysbiosis. (A and B) Principal Coordinates Analysis ( $P C O A)$ of stool microbiome at the phyla level in IAP- and vehicle-treated mice at each time point (before treatment and 6 months after treatment). (C-H) Relative abundances of different bacterial phylum, Proteobacteria (C), Actinobacteria (D), Epsilonbacteraeota (E), Deferribacteres (F), Tenericutes (C), and Verrucomicrobia $(\mathbf{H})$ in the stool of WT mice before and after supplementation with vehicle or IAP for 6 months. Each group included 6 animals. The data are averages \pm SEM. ${ }^{*} P<0.05,{ }^{* *} P<0.01,{ }^{* *} P<.001$, by a PERMANOVA and 2-way ANOVA with a Bonferroni's multiple comparisons test.

for aging studies. Drosophilae have distinct advantages in aging research, including short lifespan and ease of performing genetic manipulation. Moreover, the Drosophila genome is fully sequenced, with more than $50 \%$ of fly genes having homologs in humans (16).

We measured the kinetic profile of IAP activity in the midgut of Oregon-R (OreR) WT Drosophila (using the standard paranitrophenyl phosphate [pNPP] assay), as well as the transcript levels of 2 separate, midgut-specific alkaline phosphatase genes (CG5150 and CG10827) as a function of age. Similar to mice, we found that IAP activity and expression levels decreased significantly with age in the flies (Figure 7, A and B). To confirm the role of the 2 intestinal AP-related genes (CG5150 and CG10827) in lifespan determination, $U A S-C G 5150^{R N A i}$ and UAS-CG10827RNAi were expressed in the gut under the control of the drm-GAL4 driver. Tissue-specific knockdown of the 2 AP genes led to a significant decrease in lifespan compared with drm-GAL4 $>Y F P^{R N A i}$ control flies and double-knockdown flies supplemented with IAP (Figure 7C). Indeed, IAP supplementation was able to rescue the lifespan of double-knockdown flies. In addition, we assessed mRNA levels of 2 genes encoding important tight junctional proteins, E-cadherin and Discs Large 1 (dlgI) 
A

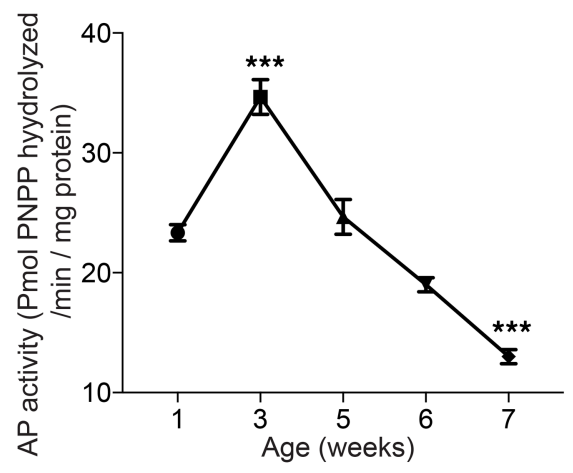

C

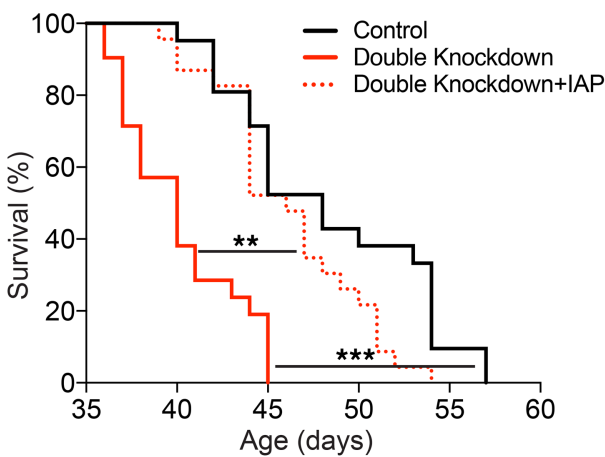

$\mathbf{E}$

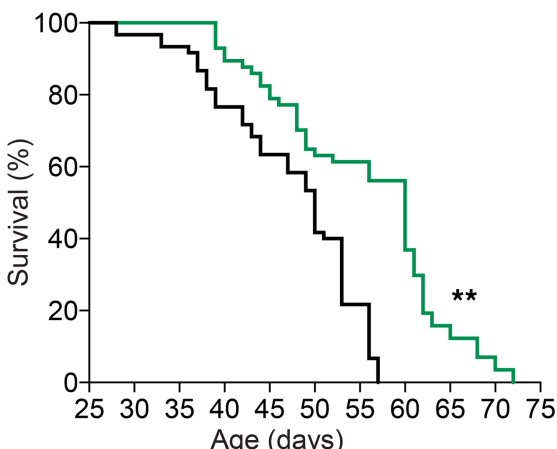

B

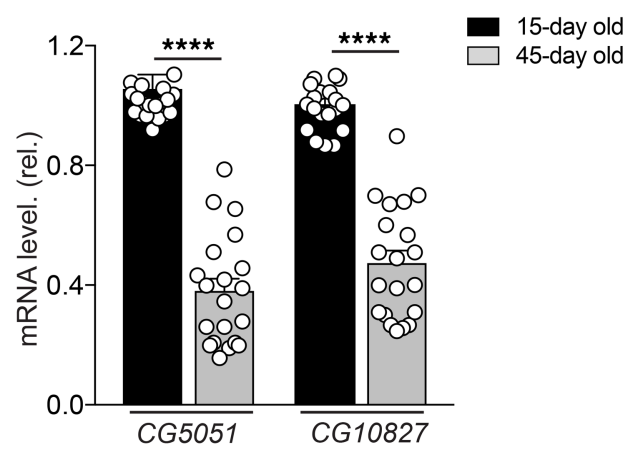

D

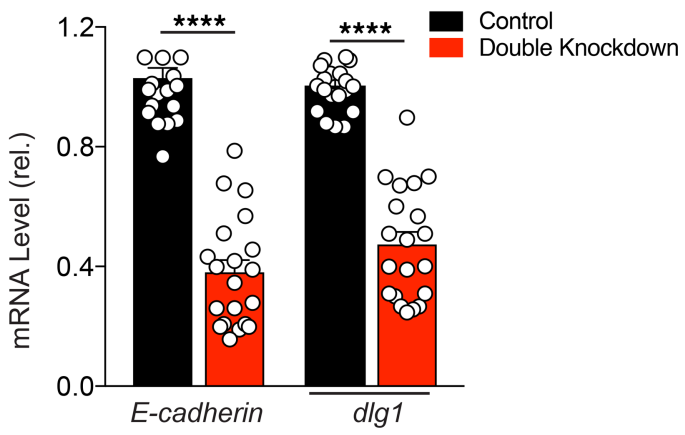

$\mathbf{F}$

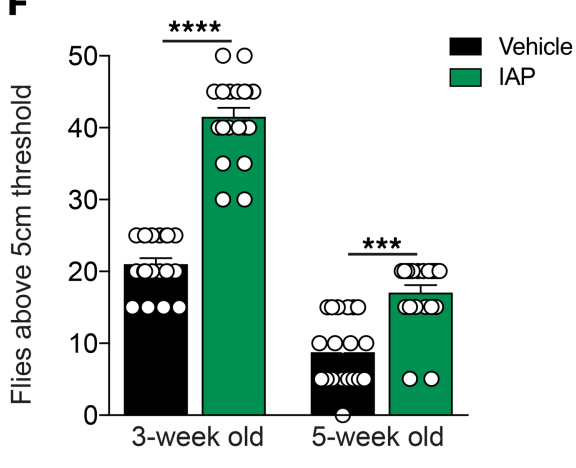

Figure 7. IAP supplementation improves physical performance and survival in Drosophilae. (A and B) AP activity and AP gene expression in the midgut of OreR Drosophilae during aging. (C) The lifespan of drm-CAL4>CG5150 ${ }^{R N A i}$, CG10827 ${ }^{\text {RAA }}$-double knockdown flies compared with drm-CAL4>YFP ${ }^{\text {RNAi }}$ control flies and double knockdown flies supplemented with IAP. (D) The mRNA levels of E-cadherin and Discs Large 1 (dlg1) in the gut of drm-GAL4>CG5150 RNAi, CG10827 ${ }^{\text {NAT }}$-knockdown flies compared with drm-GAL4>YFPRAi control flies. (E) Lifespan of WT OreR Drosophilae reared on vehicle or IAP-supplemented food. (F) The physical climbing ability of 3- and 5-week-old Drosophilae supplemented with vehicle or IAP; WT strain, Oregon-R (OreR), were used to be supplemented with IAP. Data are expressed as mean \pm SEM. Two-tailed unpaired Student's $t$ test and Kaplan-Meier curves (log-rank test) were performed as statistical tests. Results were pooled from 3 different independent experiments, with 60 flies in each group. ${ }^{*} P<0.05,{ }^{* *} P<0.01,{ }^{* *} P<0.001$.

in the gut of Drosophila, and found significantly decreased levels in AP gene double-knockdown flies as compared with control flies (Figure 7D). As with WT mice, IAP supplementation significantly increased the lifespan of WT OreR Drosophilae. Those that received exogenous IAP had a median lifespan of 60 days compared with 50 days when they received the vehicle control (Figure 7E). In another set of experiments, we found that survival was significantly prolonged when the IAP supplementation was started at 2 weeks of age; however, when started at 4 weeks of age, there was no survival benefit (data not shown). The Capillary Feeder (CAFE) assay showed that food intake was similar in flies supplemented with IAP compared with vehicle (Supplemental Figure 1). 
Aging is associated with a decline in a variety of behaviors in Drosophilae, such as negative geotaxis, an innate escape response during which flies ascend the wall of a cylinder after the bottom of the cylinder is tapped. Interestingly, we found that supplementation with exogenous IAP had a significantly positive impact on the physical climbing ability of Drosophilae (Figure 7F). Video material for a climbing assay of 3 -week-old flies receiving IAP or vehicle is available in the Supplemental Figure 1.

\section{Discussion}

Several functions of IAP such as detoxification of gut-derived proinflammatory products, maintenance of intestinal barrier integrity, and preservation of homeostasis of the gut microbiota have recently been linked to aging (8-12). A corresponding decline of IAP levels with age was observed in mice (7), but little is known about human IAP levels in aging (17). As a starting point, we confirmed a significant decrease of IAP activity with age in mice; moreover, we corroborated an age-dependent decline in humans. These findings led us to the hypothesis that IAP might affect the aging process, mainly by promoting a healthy gut barrier and through inhibition of the chronic inflammation that is thought to derive from the gut microflora. Indeed, we found that knockdown or gene deletion of IAP was associated with a shortened lifespan and aggravated frailty. Importantly, oral IAP supplementation significantly increased the lifespan and reduced frailty at different time points and in 2 different animal models. Even though IAP supplementation in mice was started rather late in life by murine standards (10 months), it was still effective in reducing frailty and metabolic deterioration. By employing different loss-of-function and supplementation animal models, we tested the role of various IAP functions connected to crucial intestinal alterations during aging.

Given the important role of the intestinal barrier in sequestering the gastrointestinal microbiome from the internal environment, disruption of this barrier during aging is associated with a variety of chronic and age-related diseases, such as metabolic syndrome, osteoarthritis, IBD, and Alzheimer's disease (16, 18-23). However, despite these numerous gut permeability-related diseases, the exact role of the intestinal barrier in aging has been poorly studied. A few studies in rodents have reported an increase in intestinal permeability with age (24-26). In tissue from aged baboons, a decrease in ZO-1 and occludin was found (27). Aged vervet monkeys exhibit reduced intestinal barrier function, leading to increased microbial translocation associated with systemic inflammation (28). Independently of aging, we have previously shown that IAP can protect the host from potentially harmful gut-derived mediators via promoting gut barrier integrity and regulation of tight junction proteins in vitro and in vivo $(9,10,15,29)$. In vitro, overexpression of IAP in intestinal epithelial cells (Caco-2 and T84) resulted in significant increases in the mRNA levels of the tight junction proteins ZO-1 and ZO-2 (9). In addition, IAP exposure ameliorated LPS-induced increased permeability in a Caco-2 cell trans-well system (9). These in vitro studies indicate that IAP, at least to some degree, promotes the gut barrier directly, independent of its inhibitory effects on inflammatory mediators and its impact on the microbial community. In the current study, we demonstrated that IAP can also prevent aging-associated gut hyperpermeability. Loss of IAP was associated with increased gut permeability and reduced expression levels of tight junction proteins, while IAP supplementation resulted in improved barrier function and reduced portal venous and systemic endotoxin levels in aging mice. The profound changes in the intestinal microbiota observed in aging may significantly contribute to compromising the intestinal barrier (1-4). The possibility of restoring a fully operational epithelial barrier either directly or via manipulation of the intestinal microbiota is a very appealing and clinically relevant therapeutic intervention.

The gut microbiome has been proposed as a possible determinant of healthy aging $(30,31)$, and the preservation of host-microbial homeostasis can counteract inflammaging, intestinal permeability, and the decline in bone and cognitive health (32-36). Consistent with this idea, a previous study found significant age-dependent differences in the fecal microbiome of young, middle-aged, and old C57BL/6J mice (37). Results of mice microbiome analysis in aging remain controversial, and prospective studies following microbial changes within the same individual across life are scarce. Most studies report microbial compositional changes with aging, and treatments targeting these age-related alterations are considered as potential approaches to prevent pathogenic deterioration and frailty. However, interventions specifically targeting gut barrier function and/or microbial composition are currently very limited. Probiotic treatment is one possible option; however, evidence of its efficacy in various disease states remains limited. Fecal microbiota transplantation was shown to be beneficial for patients with recurrent Clostridium difficile enterocolitis (38), but its efficacy in age-related disorders has not yet been tested; therefore, this approach may not be clinically practical. We have previously reported that IAP-KO mice display an overall decrease in the number and diversity 
of intestinal bacteria; furthermore, oral supplementation with IAP in WT mice was able to rapidly restore the normal gut flora in mice exposed to antibiotics (11). Therefore, in this study, we further sought to determine the effect of supplemental oral IAP on age-associated changes in commensal bacterial populations by performing 16S rRNA sequencing and analysis of fecal samples at various time points. When assessing the relative abundance of specific phyla, we observed a substantial change in phyla over a 6-month period in control mice. In contrast, IAP-treated mice displayed minimal changes in the relative abundance of phyla over time. IAP-treated mice had no significant changes in the 10 most abundant genera over 6 months of treatment. Taken together, our data demonstrate that long-term IAP treatment appears to prevent the age-associated changes in the fecal microbial composition. It is not yet clear whether the beneficial effects of IAP in the aging process are related to its impact on the composition of the gut microflora. However, based on the observations that IAP has beneficial effects using in vitro models that are independent of the flora, the mechanisms by which IAP impacts the aging process appear to be multifactorial, including its improvement in gut barrier function and its ability to inhibit bacterially derived inflammatory mediators.

LPS has been a major bacterial product linked to inflammaging (39) and plays a crucial role in various acute and chronic diseases (40). IAP is known to have the capacity to dephosphorylate and, thus, detoxify LPS $(8,41)$. The IAP enzyme primarily stays in the lumen of the gut, such that elevated AP activity can be measured in the stool of animals that receive oral IAP supplementation. Our findings confirm the importance of IAP in preventing the chronic endotoxemia that accompanies aging. Portal and systemic LPS levels increased significantly with age and reached much higher levels in IAP-KO mice. IAP supplementation in mice led to significantly decreased endotoxin/LPS levels compared with control animals. Moreover, the inflammatory potential of serum from old and IAP-KO mice, measured by target cell response, was dramatically higher compared with the serum from WT mice. In addition to the increased amounts of portal and systemic endotoxins, we found significantly higher systemic levels of the proinflammatory cytokines IL- 6 and TNF- $\alpha$. The levels of these specific cytokines have been correlated with the degree of inflammaging and age-related diseases (42). Epidemiological evidence shows that IL- 6 and TNF- $\alpha$ are predictive of many aging phenotypes and have been found to be independent risk factors for survival $(43,44)$. IL- 6 is known as the "cytokine for gerontologists," and elevated serum levels of IL- 6 are established features of aging $(45,46)$. Moreover, IL-6 has been shown to have harmful effects on intestinal permeability, with additional local and systemic implications (4). We confirmed a significant age-dependent increase in IL- 6 and TNF- $\alpha$, and we found it to be associated with more pronounced frailty in animals lacking IAP, whereas long-term IAP supplementation significantly reduced IL-6 levels and, concomitantly, frailty.

In a previous study, we have shown that IAP supplementation prevents and reverses the changes associated with a high-fat diet-induced metabolic syndrome. Furthermore, IAP improves the lipid profile during a low-fat diet (15). In the current study, we found an age-related spontaneous increase in hepatic inflammation and steatosis in mice, significantly more pronounced in mice lacking IAP. In turn, long-term IAP treatment led to lower liver enzyme and glucose levels, as well as a more favorable lipid profile in aged WT mice, underscoring the potential beneficial role of IAP in prevention of metabolic disease. Regarding the prevention of chronic inflammation and metabolic syndrome, IAP shows effects similar to caloric restriction (47). However, lifelong caloric restriction does not attenuate increased gut permeability in aging (48). It is important to note that the beneficial effects of IAP in our experiments were not due to reduced caloric intake, since there was no difference in food or water consumption or in body weight of mice receiving IAP versus vehicle $(15,29)$. In the present study, we confirmed that there were no significant differences in body weight of mice receiving long-term IAP supplementation. Similar to mice, in Drosophilae, the CAFE assay confirmed that flies supplemented with IAP consume the same amount of food as those receiving control treatment (49).

To prove the hypothesis that IAP might directly contribute to the shorter lifespan phenotype associated with tissue-specific knockdown of the 2 AP genes in Drosophilae, we supplemented these flies with IAP. Interestingly, IAP supplementation was able to rescue the lifespan of double-knockdown flies, consistent with the direct contribution of IAP in longevity. We have previously shown that IAP supplementation is beneficial in different murine models of human disease in IAP-KO mice. For example, IAP-KO mice display higher serum LPS concentrations in response to a corn oil meal compared with WT mice, and this is prevented by IAP supplementation in the IAP-KO mice (15). Moreover, in a colitis murine model, oral IAP treatment significantly reduced weight loss and inflammation severity in IAP-KO mice (50). 
In summary, our data suggest that IAP — as a natural intestinal brush border enzyme - plays a critical role in aging through maintaining intestinal microbiome homeostasis, preserving gut barrier function, and decreasing inflammaging. Future studies will focus on elucidating the precise mechanisms of this enzyme's beneficial role in aging. Given that IAP has been safely administered to humans, oral IAP supplementation could represent a novel approach to prevent a variety of aging-related diseases in humans.

Conclusion. IAP appears to preserve intestinal homeostasis in aging by targeting crucial intestinal alterations, including gut barrier dysfunction, microbiome dysbiosis, and endotoxemia. Oral IAP supplementation may represent a novel therapy to counteract the chronic inflammatory state leading to frailty and age-related diseases.

\section{Methods}

Supplemental methods are available online with this article.

Human subjects. Ileal fluid samples were collected from patients with an ileostomy and seen in the surgical clinic at the MGH. Demographics and clinical characteristics were obtained from the medical records (Supplemental Table 1).

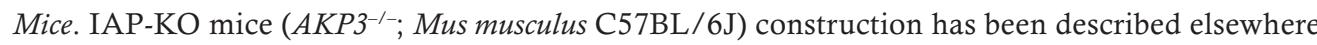
(51). Heterozygous mice were obtained from the Burnham Institute for Medical Research (La Jolla, California, USA). These specific pathogen-free (SPF) animals were subsequently bred at the MGH animal facility to create homozygous IAP-KO, heterozygous, and WT C57BL/6J littermates. Confirmation of genotype was performed by PCR analysis (51). Animals were housed in a SPF environment at MGH (Biosafety Level 2; BL2) in ventilated hard-top cages with 4 animals per cage, in accordance with the guidelines of the Committee on Animals of MGH, Harvard Medical School (Boston, Massachusetts, USA). Mice were maintained in a temperature-controlled room $\left(22^{\circ} \mathrm{C}-24^{\circ} \mathrm{C}\right)$ with a 12 -hour light/12-hour dark diurnal cycle with food and water ad libitum.

Mice experiments. Mice of different age groups - young ( $\sim 4$ months), middle age ( $\sim 12$ months), and old ( $\sim 21$ months) - and genotype (IAP-KO and WT) were used for the different experiments. The number of mice per group was at least 4, unless otherwise specified.

Lifespan of IAP-KO and WT mice. Thirty-six IAP-KO and WT mice were followed for determination of lifespan; each group contained 18 mice with the same proportion of male and female animals. Both IAP$\mathrm{KO}$ and WT mice received a regular chow diet and autoclaved tap water ad libitum. Mice were examined at least daily for health status by an experienced technician and by a member of the study group. Mice that were seriously ill and determined to likely not survive longer than 48 hours were euthanized, as per IACUC protocol. The age of the mouse at euthanasia was considered to be its natural lifespan. For mice found dead, life was calculated from the documented time within the daily controls.

IAP supplementation in WT mice. IAP supplementation was started in 10-month-old WT C57BL/6J male mice. Mice were housed in a SPF environment at MGH (BL2) as described above. WT mice received a regular chow diet and autoclaved tap water ad libitum. At the age of 10 months, 12 WT mice were examined for physical health and assigned to 2 groups. One group received IAP supplementation, and the other received vehicle. Each group contained 6 WT (Mus musculus C57BL/6J) mice (3 animals per cage). The 2 groups were treated with either bovine IAP or empty vehicle for IAP (New England Biolabs or Synthetic Biologics).

Frailty index. We quantified frailty in 60 IAP-KO and WT mice matched for sex and age using the performance-based frailty index, which is a noninvasive clinical assessment and contains key features of the frailty index established for use in humans. This clinical assessment included evaluation of the integument, the musculoskeletal system, the ocular and nasal systems, the digestive system, the urogenital system, the respiratory system, signs of discomfort, the body weight, and body surface temperature (52). In addition, the same index was measured at different time points in 12 male WT mice before and during IAP treatment throughout the experimental period.

IAP assay. The IAP assay has been previously described and can be found in Supplemental methods (10-12).

Fecal DNA extraction and $16 S$ rRNA sequencing. Fresh fecal samples were collected from mice before starting IAP supplementation and again at 1- and 6-month time points after supplementation.

Drosophilae. The WT strain, OreR, was used to measure IAP activity in the midgut of aging flies. Midguts from 20 WT OreR flies of the indicated ages were dissected in 1× PBS, and total RNA was extracted using the RNeasy Plus kit (Qiagen), followed by reverse transcription using the iScript Kit (Bio-Rad), according to the manufacturer's protocol. The resulting cDNA was used as a template for quantitative PCR (qPCR) 
using the SYBR Green qPCR low ROX $2 \times$ Mastermix (Agilent) to quantify the expression of the various midgut-specific alkaline phosphatase genes normalized to $r p 49$ as an internal control. For each sample, qPCR reactions were performed in triplicate. Primer sequences are provided in the Supplemental Table 3.

For obtaining fly lines with knockdown of the 2 AP genes CG5150 (location, chromosome 3L, 64D5-64D5 [+strand], 5,595,542-5,597,422) and CG10827 (location, chromosome 3R, 64D5-64D5 [-strand], $21,005,160-21,006,990)$ in the midgut, the drumstick-GAL4/Tm3 (drm-GAL4/Tm3) (gift from Robert Salomon, Tufts Medical Center, Boston, Massachusetts, USA), UAS-CG5150 RNAi /CyO (TRiP HMC04450), and $U A S-C G 10827^{R N A i}$ (TRiP HMC04841) strains were first isogenized by backcrossing for 8 generations to the $\mathrm{y}^{1} \mathrm{~W}^{67 \mathrm{c} 23} \mathrm{WT}$ background in order to generate isogenic lines. The UAS-YFPRAAi line was used as the control RNAi. First, UAS-CG5150 RNAi /CyO; drm-GAL4/Tm3 was generated and then crossed to UAS-CG10827RNAi to yield the UAS-CG5150 RNAi /CG10827 ${ }^{\mathrm{RNAi}}$; $d r m-G A L 4 /+$ flies. For control flies, $d r m-G A L 4 / T m 3$ was crossed to $U A S-Y F P^{R N A i}$ to yield the $U A S-Y F P^{R N A i} /+; d r m-G A L 4 /+$ flies.

Survival kinetics were evaluated daily. IAP supplementation assay was conducted by providing the equivalent 1 unit/fly per day in regular food. The number of flies for each survival experiment is provided in Supplemental Table 2. The climbing assay was conducted by tapping down 20 flies and quantifying the percentage of them that climbed above the $5-\mathrm{cm}$ threshold after 15 seconds. For assessing mRNA levels of junction-related genes, midguts were dissected and qPCR was conducted as described above. All fly experiments were performed in 3 replicates and in both male and female flies.

Statistics. All statistical calculations were performed using Prism 7 (GraphPad software). Duplicate or triplicate samples were used for all assays. All experiments were repeated 3 times, and data were analyzed using Student's $t$ test ( 2 tailed) or 2-way ANOVA with multiple-comparisons post hoc tests, as appropriate. For survival analyses, Kaplan-Meier curves were generated and compared using the log-rank significance test. Survival data were compared using the log-rank significance test.

$\beta$-Diversity (between-sample diversity) was determined through principle coordinates analysis (PCoA) plots of weight UniFrac distance and the Kruskal Walls test.

For microbiome analysis, the Kruskal-Wallis test was used as an exploratory tool to look for potential differences across time points in each treatment group. $P$ values $\left({ }^{*} P<0.05 ;{ }^{*} P<0.01 ;{ }^{* *} P<0.001\right.$ : $* * * * P<0.0001$ ) were considered statistically significant using a PERMANOVA and 2-way ANOVA with a Bonferroni's multiple comparisons test.

Study approval. For human studies, all participants provided written informed consent, and the study was approved by the MGH IRB (protocol no. 2011P002755). All animal protocols were reviewed and approved by the IACUC and according to regulations of the Subcommittee on Research Animal Care of the MGH and the National Institutes of Health.

\section{Author contributions}

FK designed research, conducted experiments, acquired data, analyzed data, and wrote the manuscript. FA designed research, conducted experiments, acquired data, analyzed data, performed statistical analyses, and wrote the manuscript. PMC, SRH, AT, RSH, JMR, ARM, EL, RV, YL, ES, RAN, $\mathrm{MZF}$, and VC acquired data and conducted experiments. MSM, LGR, and RAH designed research, analyzed data, and critically revised the manuscript for intellectual content. All authors revised and approved the manuscript for publication.

\section{Acknowledgments}

This work was supported by grants from the Ellison Foundation and the Nutrition Obesity Research Center at Harvard (NORCH, NIH P30-DK040561) to RAH; FK was supported by a research fellowship granted from the German Research Foundation (DFG), grant no. KU 3464/2-1. We thank the TRiP at Harvard Medical School (NIH/NIGMS R01-GM084947) for providing transgenic RNAi fly stocks used in this study. We thank Duy Dinh, Lucia Martini, and Emily Hollister (Diversigen Inc.) for assistance with microbiome analysis.

Address correspondence to: Richard A. Hodin, Harvard Medical School, Department of Surgery, Massachusetts General Hospital, 15 Parkman Street, Boston, Massachusetts 02114, USA. Phone: 617.724.2570; Email: rhodin@mgh.harvard.edu. 
1. An R, Wilms E, Masclee AAM, Smidt H, Zoetendal EG, Jonkers D. Age-dependent changes in GI physiology and microbiota: time to reconsider? Gut. 2018;67(12):2213-2222.

2. Kundu P, Blacher E, Elinav E, Pettersson S. Our Gut Microbiome: The Evolving Inner Self. Cell. 2017;171(7):1481-1493.

3. Thevaranjan N, et al. Age-Associated Microbial Dysbiosis Promotes Intestinal Permeability, Systemic Inflammation, and Macrophage Dysfunction. Cell Host Microbe. 2017;21(4):455-466.e4.

4. Nicoletti C. Age-associated changes of the intestinal epithelial barrier: local and systemic implications. Expert Rev Gastroenterol Hepatol. 2015;9(12):1467-1469.

5. Franceschi C, Campisi J. Chronic inflammation (inflammaging) and its potential contribution to age-associated diseases. $J$ Gerontol $A$ Biol Sci Med Sci. 2014;69 Suppl 1:S4-S9.

6. Goldstein DJ, Rogers CE, Harris H. Expression of alkaline phosphatase loci in mammalian tissues. Proc Natl Acad Sci USA. 1980;77(5):2857-2860

7. Detel D, Baticic L, Varljen J. The influence of age on intestinal dipeptidyl peptidase IV (DPP IV/CD26), disaccharidases, and alkaline phosphatase enzyme activity in C57BL/6 mice. Exp Aging Res. 2008;34(1):49-62.

8. Chen KT, et al. Identification of specific targets for the gut mucosal defense factor intestinal alkaline phosphatase. Am J Physiol Gastrointest Liver Physiol. 2010;299(2):G467-G475.

9. Liu W, et al. Intestinal Alkaline Phosphatase Regulates Tight Junction Protein Levels. J Am Coll Surg. 2016;222(6):1009-1017.

10. Hamarneh SR, et al. A novel approach to maintain gut mucosal integrity using an oral enzyme supplement. Ann Surg. 2014;260(4):706-714.

11. Malo MS, et al. Intestinal alkaline phosphatase preserves the normal homeostasis of gut microbiota. Gut. 2010;59(11):1476-1484

12. Malo MS, et al. Intestinal alkaline phosphatase promotes gut bacterial growth by reducing the concentration of luminal nucleotide triphosphates. Am J Physiol Gastrointest Liver Physiol. 2014;306(10):G826-G838

13. Pickkers P, et al. Alkaline phosphatase for treatment of sepsis-induced acute kidney injury: a prospective randomized double-blind placebo-controlled trial. Crit Care. 2012;16(1):R14.

14. Lukas M, et al. Exogenous alkaline phosphatase for the treatment of patients with moderate to severe ulcerative colitis. Inflamm Bowel Dis. 2010;16(7):1180-1186.

15. Kaliannan K, et al. Intestinal alkaline phosphatase prevents metabolic syndrome in mice. Proc Natl Acad Sci USA. 2013;110(17):7003-7008.

16. Camilleri M, Madsen K, Spiller R, Greenwood-Van Meerveld B, Van Meerveld BG, Verne GN. Intestinal barrier function in health and gastrointestinal disease. Neurogastroenterol Motil. 2012;24(6):503-512.

17. Malo MS. A High Level of Intestinal Alkaline Phosphatase Is Protective Against Type 2 Diabetes Mellitus Irrespective of Obesity. EBioMedicine. 2015;2(12):2016-2023.

18. Teshima CW, Dieleman LA, Meddings JB. Abnormal intestinal permeability in Crohn's disease pathogenesis. Ann N Y Acad Sci. 2012;1258:159-165.

19. Büning C, et al. Increased small intestinal permeability in ulcerative colitis: rather genetic than environmental and a risk factor for extensive disease? Inflamm Bowel Dis. 2012;18(10):1932-1939.

20. Teixeira TF, et al. Intestinal permeability parameters in obese patients are correlated with metabolic syndrome risk factors. Clin Nutr. 2012;31(5):735-740.

21. Fasano A. Leaky gut and autoimmune diseases. Clin Rev Allergy Immunol. 2012;42(1):71-78.

22. Leblhuber F, Geisler S, Steiner K, Fuchs D, Schütz B. Elevated fecal calprotectin in patients with Alzheimer's dementia indicates leaky gut. J Neural Transm (Vienna). 2015;122(9):1319-1322.

23. Turner JR. Intestinal mucosal barrier function in health and disease. Nat Rev Immunol. 2009;9(11):799-809.

24. Hollander D, Tarnawski H. Aging-associated increase in intestinal absorption of macromolecules. Gerontology. 1985;31(3):133-137.

25. Katz D, Hollander D, Said HM, Dadufalza V. Aging-associated increase in intestinal permeability to polyethylene glycol 900. Dig Dis Sci. 1987;32(3):285-288.

26. Annaert P, Brouwers J, Bijnens A, Lammert F, Tack J, Augustijns P. Ex vivo permeability experiments in excised rat intestinal tissue and in vitro solubility measurements in aspirated human intestinal fluids support age-dependent oral drug absorption. Eur J Pharm Sci. 2010;39(1-3):15-22.

27. Tran L, Greenwood-Van Meerveld B. Age-associated remodeling of the intestinal epithelial barrier. J Gerontol A Biol Sci Med Sci. 2013;68(9):1045-1056.

28. Mitchell EL, et al. Reduced Intestinal Motility, Mucosal Barrier Function, and Inflammation in Aged Monkeys. J Nutr Health Aging. 2017;21(4):354-361.

29. Hamarneh SR, et al. Intestinal Alkaline Phosphatase Attenuates Alcohol-Induced Hepatosteatosis in Mice. Dig Dis Sci. 2017;62(8):2021-2034.

30. Biagi E, Candela M, Franceschi C, Brigidi P. The aging gut microbiota: new perspectives. Ageing Res Rev. 2011;10(4):428-429.

31. Nagpal R, et al. Gut microbiota in health and disease: an overview focused on metabolic inflammation. Benef Microbes. 2016;7(2):181-194.

32. Claesson MJ, et al. Gut microbiota composition correlates with diet and health in the elderly. Nature. 2012;488(7410):178-184

33. Candela M, Biagi E, Brigidi P, O'Toole PW, De Vos WM. Maintenance of a healthy trajectory of the intestinal microbiome during aging: a dietary approach. Mech Ageing Dev. 2014;136-137:70-75.

34. Villa CR, Ward WE, Comelli EM. Gut microbiota-bone axis. Crit Rev Food Sci Nutr. 2017;57(8):1664-1672.

35. Leung K, Thuret S. Gut Microbiota: A Modulator of Brain Plasticity and Cognitive Function in Ageing. Healthcare (Basel). 2015;3(4):898-916.

36. Singh R, et al. Enhancement of the gut barrier integrity by a microbial metabolite through the Nrf2 pathway. Nat Commun 2019;10(1):89.

37. Langille MG, et al. Microbial shifts in the aging mouse gut. Microbiome. 2014;2(1):50.

38. van Nood E, et al. Duodenal infusion of donor feces for recurrent Clostridium difficile. N Engl J Med. 2013;368(5):407-415.

39. Kim KA, Jeong JJ, Yoo SY, Kim DH. Gut microbiota lipopolysaccharide accelerates inflamm-aging in mice. BMC Microbiol. 2016;16:9. 
40. Guerville M, Boudry G. Gastrointestinal and hepatic mechanisms limiting entry and dissemination of lipopolysaccharide into the systemic circulation. Am J Physiol Gastrointest Liver Physiol. 2016;311(1):G1-G15.

41. Estaki M, DeCoffe D, Gibson DL. Interplay between intestinal alkaline phosphatase, diet, gut microbes and immunity. World J Gastroenterol. 2014;20(42):15650-15656.

42. Minciullo PL, et al. Inflammaging and Anti-Inflammaging: The Role of Cytokines in Extreme Longevity. Arch Immunol Ther $\operatorname{Exp}$ (Warsz). 2016;64(2):111-126.

43. Varadhan R, et al. Simple biologically informed inflammatory index of two serum cytokines predicts 10 year all-cause mortality in older adults. J Gerontol A Biol Sci Med Sci. 2014;69(2):165-173.

44. Ershler WB. Interleukin-6: a cytokine for gerontologists. J Am Geriatr Soc. 1993;41(2):176-181.

45. Giovannini S, et al. Interleukin-6, C-reactive protein, and tumor necrosis factor-alpha as predictors of mortality in frail, community-living elderly individuals. J Am Geriatr Soc. 2011;59(9):1679-1685.

46. Michaud M, et al. Proinflammatory cytokines, aging, and age-related diseases. J Am Med Dir Assoc. 2013;14(12):877-882.

47. Mattison JA, et al. Caloric restriction improves health and survival of rhesus monkeys. Nat Commun. 2017;8:14063.

48. Parrish AR. The impact of aging on epithelial barriers. Tissue Barriers. 2017;5(4):e1343172.

49. Diegelmann S, et al. The CApillary FEeder Assay Measures Food Intake in Drosophila melanogaster. J Vis Exp. 2017;(121):55024

50. Ramasamy S, et al. Intestinal alkaline phosphatase has beneficial effects in mouse models of chronic colitis. Inflamm Bowel Dis. 2011;17(2):532-542.

51. Narisawa S, Huang L, Iwasaki A, Hasegawa H, Alpers DH, Millán JL. Accelerated fat absorption in intestinal alkaline phosphatase knockout mice. Mol Cell Biol. 2003;23(21):7525-7530.

52. Whitehead JC, et al. A clinical frailty index in aging mice: comparisons with frailty index data in humans. J Gerontol A Biol Sci Med Sci. 2014;69(6):621-632. 